\title{
Factors Associated with the Effectiveness of National Free Antiretroviral Treatment Program in HIV Infected Patients of Yi Nationality from Southwest China: Evidence from A Cohort Study to Provide Clues for Improvement
}

\section{Liyu Chen}

west china hospital, sichuan university

\section{Shuang Kang}

Sichuan University West China Hospital https://orcid.org/0000-0002-8390-7665

\section{Lingyao Du}

west china hospital

\section{Fanghua Ma}

People's hospital of Zhaojue county

Changmin Li

People's hospital of Zhaojue county

\section{Lang Bai}

West China hospital of Sichuan University

Hong Tang ( $\sim$ htang6198@hotmail.com)

west china hospital, sichuan university

\section{Research}

Keywords: HIV/AIDS, antiretroviral treatment, virologic suppression, low level viremia, immune recovery

Posted Date: November 6th, 2020

DOl: https://doi.org/10.21203/rs.3.rs-98257/v1

License: (c) (1) This work is licensed under a Creative Commons Attribution 4.0 International License.

Read Full License 
1 Factors associated with the effectiveness of National Free Antiretroviral

2 Treatment Program in HIV infected patients of Yi nationality from southwest

3 China: evidence from a cohort study to provide clues for improvement

4 Liyu Chen ${ }^{1,2 \#}$, Shuang Kang ${ }^{1,3 \#}$, Lingyao $\mathrm{Du}^{1}$, Fanghua $\mathrm{Ma}^{2}$, Changmin $\mathrm{Li}^{2}$, Lang Bai ${ }^{1}$, Hong Tang ${ }^{1,3 *}$ 5

6 Affiliations:

7 1. Center of Infectious Diseases, West China Hospital of Sichuan University, Chengdu, China, $8610041 ;$

9 2. Center of antiretroviral treatment, People's Hospital of Zhaojue County, Liangshan Yi 10 Autonomous Prefecture, China, 616150;

11 3. Division of Infectious Diseases, State Key Laboratory of Biotherapy and Center of Infectious

12 Diseases, West China Hospital, Sichuan University, Chengdu, China, 610041.

13 \# The first two authors contributed equally to the manuscript.

14 * Correspondence:

15 Hong Tang

16 htang6198@hotmail.com

17 
Abstract:

Background: Despite the current achievements of HIV management in the source-limited region of Liangshan Autonomous Prefecture, a small population remains with unsatisfactory virologic outcomes and suboptimal immune recovery. This cohort study aimed to identify potential risk factors of suboptimal clinical outcomes and to provide clues for improvement in people living with HIV (PLWH) from China's National Free Antiretroviral Treatment Program (NFATP) in Zhaojue County, Liangshan Autonomous Prefecture of Yi nationality.

Results: A total of 608 HIV infected adult patients in NFATP at the median age of $35(31,40)$ were enrolled for analysis. During the 2.15(1.54, 4.00)-year follow-up, $502(82.6 \%)$ patients achieved the viral load of $<1000$ copies $/ \mathrm{mL}$ after over 6 months of antiretroviral therapy(ART). Among them, $398(65.5 \%)$ cases achieved complete viral suppression with viral load $<50$ copies $/ \mathrm{mL}$ while 104 (17.1\%) cases remained with low level viremia (LLV, 50 $\leq$ viral load $\leq 1000$ copies $/ \mathrm{mL}$ ). Patients with longer infection duration ( $\mathrm{OR}=1.017$ [95\%CI: 1.002-1.033], $p=0.026)$, male gender (OR $=1.632$ [95\%CI: 1.053-2.53], $p=0.028)$, positive hepatitis $\mathrm{C}$ virus antibody $(\mathrm{OR}=1.687$ [95\%CI: $1.093-2.604], p=0.018)$ or infection through injecting drug use (IDU) (OR $=1.584$ [95\%CI: 1.022 2.455], $p=0.04$ ) were more likely to experience the undesirable outcome of LLV. Moreover, 254 (63.8\%) of 398 patients with viral suppression achieved optimal immune recovery with the CD4 count $\geq 350$ cells $/ \mu \mathrm{L}$. Patients with lower body mass index (BMI) $\left(21.00 \pm 2.94 \mathrm{Kg} / \mathrm{m}^{2}, \mathrm{OR}=0.921\right.$ [95\%CI: 0.854-0.994], $p=0.034)$ and higher fasting blood glucose $(4.99 \pm 1.01 \mathrm{mmol} / \mathrm{L}, \mathrm{OR}=1.343$ [95\%CI: $1.087-1.658], p=0.006)$ were less likely to achieve the CD4 count $\geq 350$ cells $/ \mu \mathrm{L}$ after viral suppression.

Conclusions: In this long-term cohort study of PLWH from Zhaojue County, Liangshan, most patients achieved virologic success on ART provided by NFATP but optimal immune recovery was yet to be expected. Approaches including strict control of illegal drug deal, patient education, and nutritional status management could be conducive to better prognosis for this population. immune recovery. 


\section{Background:}

Human immunodeficiency virus (HIV) infection has been one of the major global challenges since 1981. Although effective ART has transformed HIV infection from a death sentence to a chronic controllable condition, certain intractable issues remain and a subset of the PLWH fail to achieve the expected recovery under current ART. It's of great urgency and importance to unravel the underlying cause to improve the treatment effectiveness in this population. At the end of 2019, there were approximately 958,000 PLWH in China. The dominant transmission route is heterosexual activities with a proportion of $71.5 \%$, followed by homosexual of $23.3 \%$ and transmission by IDU has declined substantially to the level of 3\% in 2018(1). Massive endeavor has been made for HIV/AIDS prevention and control, such as the NFATP initiated in 2002 and the rigorous implementation of "Five Expansions and Six Strengthens" since 2011. The overall treatment coverage increased to $83.4 \%$ at the end of $2018(2)$ and $89 \%$ of the PLWH with a viral load test in 2015 achieved viral suppression(3).

There is substantial regional variation across China in HIV epidemic and Liangshan is one of the areas with the highest HIV prevalence and very unique epidemics. It is a remote, impoverished district in southwest of China domiciled mostly with ethnic minority people of Yi nationality. Despite the relatively smaller population, people of Yi nationality accounts for $88.07 \%$ of new HIV infections from 2011-2013(4). The HIV prevalence rate in 5 counties of Liangshan was the highest across China(4) with an cumulative mortality rate of 10.02\% during 2005-2015(5). The dominant transmission routes are heterosexual behaviors and IDU in Liangshan Prefecture, distinct from other regions in China. Multiple factors have given rise to the current heavy disease burden and management difficulties, including poverty, casual sexual culture, unique marriage practice and prior history of rampant drug transition. The rate of complete virologic suppression is reported as $58.2 \%$ in 2017(6), lower than that in other provinces in China. Evidence is urgently warranted to disclose the underlying problems and provide clues for further improvement. Nevertheless, there is

71 a paucity of literature concerning the comprehensive medical characteristics of PLWH in this area recently, with most cross-sectional studies focused on the social and humanistic or epidemiological aspects. Zhaojue county is among the areas with highest HIV prevalence in Liangshan and is the 
model county of NFATP with relatively complete records of patients. Thus, this study is aimed to

retrospectively establish a cohort of HIV infected patients under regular ART and to assess the

treatment effectiveness and identify risk factors of unsatisfactory immune recovery.

\section{Results:}

\section{Demographics}

A total of 608 patients in Yi nationality at the median age of $35(31,40)$ years old were enrolled in the cohort. The majority of patients $(84.0 \%)$ were sexually active as married or cohabiting with their partners. IDU (52.1\%) and heterosexual behaviors (46.1\%) are the two dominant routes of infection, which is exemplary of the unique HIV epidemiology in this area. At ART initiation, the median infection duration was about 1 year with a high HIV VL of $5.21(5.01,5.35) \log _{10} \operatorname{copies} / \mathrm{mL}$ indicating active viral replication but most patients $(486,79.9 \%)$ showed relatively good immune state with the CD4+ counts $\geq 350$ cells $/ \mu \mathrm{L}$. It was worth attention that the HBV- and HCVcoinfection were strikingly serious in this cohort. According to previous surveys in the general Chinese population, the prevalence of HBV surface antigen (HBsAg) is $7.2 \%(7)$ and that of $\mathrm{HCV}$ antibody $1.0-2.9 \%(8)$. PLWH are at higher risk of HBV and HCV infection with HIV-HBV coinfection rate of $9.5 \%$ and HIV-HCV $8.3 \%$ in a study including PLWH from 12 provinces across 5 regions in China(9). The prevalence of HBsAg (11.3\%) and HCV antibody (46.5\%) in this cohort were both much higher than the general population as well as the overall PLWH across China, which might be attributed to the IDU-dominated transmission. As for initial ART regimens, $97.2 \%$ patients preferred choice of NNRTIs.

Table 1. Baseline characteristics of study subjects

\begin{tabular}{ll}
\hline \multicolumn{2}{c}{$\mathbf{N}=\mathbf{6 0 8}$} \\
\hline $\begin{array}{l}\text { Gender (cases, ratio) } \\
\text { Male }\end{array}$ & $322(53.0 \%)$ \\
Age(years) & $35(31,40)$ \\
Marital status (cases, ratio) & \\
$\quad$ Married/cohabiting & $511(84.0 \%)$ \\
Single/divorced/widowed & $74(12.2 \%)$ \\
Others & $13(2.1 \%)$ \\
Routes of infection & $317(52.1 \%)$ \\
IDU & $280(46.1 \%)$ \\
Heterosexual contact & $2(0.3 \%)$ \\
Homosexual contact & $2(0.3 \%)$ \\
Vertical transmission &
\end{tabular}




\begin{tabular}{|c|c|}
\hline Others & $5(0.8 \%)$ \\
\hline $\begin{array}{l}\text { HIV VL } \\
(\text { copies/mL) }\end{array}$ & $5.21(5.01,5.35)$ \\
\hline $\begin{array}{l}\text { CD4+ count } \\
(\text { cell } / \mu \mathrm{L})\end{array}$ & $364(230,528)$ \\
\hline$<200$ & $20(3.3 \%)$ \\
\hline $200 \leq \mathrm{CD} 4+<350$ & $102(16.8 \%)$ \\
\hline $350 \leq \mathrm{CD} 4+<500$ & $304(50 \%)$ \\
\hline$>500$ & $182(29.9 \%)$ \\
\hline $\begin{array}{l}\text { Infection duration } \\
\text { (years) }\end{array}$ & $1.00(0.25,2.17)$ \\
\hline \multicolumn{2}{|l|}{ ART regimen } \\
\hline $\mathrm{AZT}+3 \mathrm{TC}+\mathrm{EFV}$ & $182(29.9 \%)$ \\
\hline $\mathrm{AZT}+3 \mathrm{TC}+\mathrm{NVP}$ & $20(3.3 \%)$ \\
\hline $\mathrm{TDF}+3 \mathrm{TC}+\mathrm{EFV}$ & $376(61.8 \%)$ \\
\hline $\mathrm{TDF}+3 \mathrm{TC}+\mathrm{NVP}$ & $11(\%)$ \\
\hline $\mathrm{AZT} / \mathrm{TDF}+3 \mathrm{TC}+\mathrm{LPV} / \mathrm{R}$ & $17(2.8 \%)$ \\
\hline HBV coinfection & $69(11.3 \%)$ \\
\hline HCV coinfection & $283(46.5 \%)$ \\
\hline
\end{tabular}

All statistics are shown as median (IQR) or cases (percent).

Virologic treatment efficacy and associated factors

Till the end of $2.15(1.54,4.00)$-year follow-up, $502(82.6 \%)$ patients achieved virologic

treatment success with the $\mathrm{VL}<1000$ copies/mL after 6 months of ART among whom 398 (65.5\%)

cases achieved complete viral suppression with $\mathrm{VL}<50$ copies $/ \mathrm{mL}$, indicating the relatively satisfying treatment effectiveness. At ART initiation, the patients across three subgroups were with comparable HIV viral load and CD4 cell counts. But $16.9 \%$ of the patients experienced LLV and

$103 \quad 17.6 \%$ of the patients were observed with viral failure. The male patients were overrepresented in

104 the subgroup with $\operatorname{LLV}(58.7 \%)$ or viral failure (67.9\%) compared with the subgroup with vial 105 suppression $(47.5 \%, p<0.001)$. The duration of infection was significantly longer and the duration 106 of ART was significantly shorter in the subgroup with suboptimal virologic outcomes (LLV or viral 107 failure) compared with the subgroup with vial suppression as shown in Table. Besides, there were 108 substantially more patients with positive HCV Ab in the LLV or viral failure group ( $51.9 \%$ vs. $10966.0 \%$ vs. $39.9 \%$, respectively, $p<0.001)$.

110 For patients with viral failure, drug-resistant mutation detection and change of ART regimen 111 are recommended while the management of patients with LLV has not yet reached a consensus. It's 112 generally recommended to monitor these patients more closely for potential clinical evolutions, and 113 possible interventions other than regimen modification might be implemented at the same time. 114 Thus, comparison between the subgroups with viral suppression and LLV was conducted with 
115 univariate analysis, aiming to identify potential intervention target for better outcomes. The results

116 unraveled several risk factors for LLV including longer infection duration (OR $=1.017$ [95\%CI:

117 1.002-1.033], $p=0.026$ ), male gender ( $\mathrm{OR}=1.632$ [95\%CI: 1.053-2.53], $p=0.028)$, positive $\mathrm{HCV}$

$118 \mathrm{Ab}(\mathrm{OR}=1.687$ [95\%CI: 1.093-2.604], $p=0.018)$ and infection through IDU (OR $=1.584$ [95\%CI:

119 1.022-2.455], $p=0.04)$. Although multivariate analysis failed to reveal any risk factors with

120 statistical significance, it could be implied that enhanced screening to early detect HIV infected

121 patients, rigorous actions to control illegal drug dealing and strengthened supervision on male

122 patients to improve treatment compliance might provide certain benefits for PLWH in this area.

Table 2. Subject characteristics by virologic outcomes

\begin{tabular}{|c|c|c|c|c|}
\hline & Viral suppression & $\mathbf{L L V}$ & Viral failure & \\
\hline $\mathbf{N}$ & $398(65.5 \%)$ & $104(17.1 \%)$ & $106(17.4 \%)$ & \\
\hline \multicolumn{5}{|l|}{$\begin{array}{l}\text { Gender } \\
\text { (cases, ratio) }\end{array}$} \\
\hline Male & $189(47.5)$ & $61(58.7 \%)$ & $72(67.9 \%)$ & $<0.001$ \\
\hline Age(years) & $35(31-41)$ & $35(30-40)$ & $36(31-40)$ & \\
\hline \multicolumn{5}{|l|}{$\begin{array}{l}\text { Marital status } \\
\text { (cases, ratio) }\end{array}$} \\
\hline Married/cohabiting & $339(85.2 \%)$ & $89(85.6 \%)$ & $93(85.6 \%)$ & \\
\hline Single/divorced/widowed & $48(12.1 \%)$ & $15(14.4 \%)$ & $11(12.5 \%)$ & \\
\hline Others & $11(2.8 \%)$ & 0 & $2(1.9 \%)$ & \\
\hline \multicolumn{5}{|l|}{ Routes of infection } \\
\hline IDU & $192(48.2 \%)$ & $62(59.6 \%)$ & $63(59.4 \%)$ & \\
\hline Heterosexual contact & $200(50.3 \%)$ & $37(35.6 \%)$ & $43(40.6 \%)$ & \\
\hline Homosexual contact & $2(0.5 \%)$ & 0 & 0 & \\
\hline Vertical transmission & $1(0.3 \%)$ & 0 & 0 & \\
\hline Others & $3(0.8 \%)$ & $5(2.0)$ & 0 & \\
\hline $\begin{array}{l}\text { Baseline HIV VL } \\
\text { (copies/ML) }\end{array}$ & $5.21(5.03-5.36)$ & $5.24(4.99-5.36)$ & $5.16(4.92-5.34)$ & \\
\hline $\begin{array}{l}\text { Baseline CD4+ count } \\
(\text { cell } / \mu \mathrm{L})\end{array}$ & $369(234-531)$ & $336(204-536)$ & $364(219-505)$ & \\
\hline$<200$ & $75(18.8 \%)$ & $23(22.1 \%)$ & $24(22.6 \%)$ & \\
\hline $200 \leq \mathrm{CD} 4+<350$ & $115(28.9 \%)$ & $32(30.8 \%)$ & $27(25.5 \%)$ & \\
\hline $350 \leq \mathrm{CD} 4+<500$ & $87(21.9 \%)$ & $18(17.3 \%)$ & $25(23.6 \%)$ & \\
\hline$>500$ & $121(30.4)$ & $31(29.8 \%)$ & $30(28.3 \%)$ & \\
\hline $\begin{array}{l}\text { Endpoint CD4+ count } \\
(\text { cell } / \mu \mathrm{L})\end{array}$ & $402(278,598)$ & $365(235,472)$ & $251(166,401)$ & $<0.001$ \\
\hline $\begin{array}{l}\text { Infection duration } \\
\text { (years) }\end{array}$ & $4.13(3.25-4.75)$ & $4.25(3.31-4.92)$ & $4.29(3.08-5.08)$ & $<0.001$ \\
\hline $\begin{array}{l}\text { ART duration } \\
\text { (years) }\end{array}$ & $2.43(1.66-4.00)$ & $2.01(1.40-4.02)$ & $2.02(1.38-3.41)$ & 0.008 \\
\hline \multicolumn{5}{|l|}{ Initial ART Regimen } \\
\hline $\mathrm{AZT}+3 \mathrm{TC}+\mathrm{EFV}$ & $109(27.4 \%)$ & $34(32.7 \%)$ & $39(36.8 \%)$ & \\
\hline $\mathrm{AZT}+3 \mathrm{TC}+\mathrm{NVP}$ & $252(63.3 \%)$ & $62(59.6 \%)$ & $62(58.5 \%)$ & \\
\hline $\mathrm{TDF}+3 \mathrm{TC}+\mathrm{EFV}$ & $15(3.8 \%)$ & $2(1.9 \%)$ & $3(2.8 \%)$ & \\
\hline $\mathrm{TDF}+3 \mathrm{TC}+\mathrm{NVP}$ & $8(2 \%)$ & $2(1.9 \%)$ & $1(0.9 \%)$ & \\
\hline $\mathrm{AZT} / \mathrm{TDF}+3 \mathrm{TC}+\mathrm{LPV} / \mathrm{r}$ & $14(3.5 \%)$ & $4(1.6 \%)$ & $1(0.9 \%)$ & \\
\hline HBV coinfection & $40(10.1 \%)$ & $16(15.3 \%)$ & $13(12.3 \%)$ & \\
\hline HCV coinfection & $159(39.9 \%)$ & $54(51.9 \%)$ & $70(66.0 \%)$ & $<0.001$ \\
\hline
\end{tabular}


125 Despite the relatively satisfying virologic outcomes, only 254 (63.8\%) of the 398 patients with viral

126 suppression achieved optimal immune recovery with the CD4 counts $\geq 350$ cells $/ \mu \mathrm{L}$. The

127 approximate 2-year follow-up may be inadequate to determine immune non-responder since the

128 increase of CD4 count could span more than 5 years. But early identification of relevant risk factors

129 and timely intervention could potentially bring additional benefits. Therefore, logistic regression

130 analysis was conducted. Since more and more studies have been looking into the metabolic diseases

131 in PLWH and have found certain evidence indicating the association of metabolic profiles and ART

132 outcomes, baseline metabolic profiles including BMI, fasting blood glucose, and blood lipids were

133 also included in the analysis. All the patients in this subgroup analysis displayed a metabolic profile

134 within normal ranges (data not shown). The risk factors significantly related to suboptimal immune

135 recovery identified by multivariate analysis include baseline $\mathrm{CD} 4$ count $(\mathrm{OR}=1.000,[95 \% \mathrm{CI}$ :

$1361.000-1.000], p=0.004)$, BMI $\left(21.00 \pm 1.94 \mathrm{Kg} / \mathrm{m}^{2}, \mathrm{OR}=0.921\right.$ [95\%CI: 0.854-0.994], $\left.p=0.034\right)$

137 and fasting blood glucose $(4.99 \pm 1.01 \mathrm{mmol} / \mathrm{L}, \mathrm{OR}=1.343$ [95\%CI: 1.087-1.658], $p=0.006)$. After

138 achieving viral suppression, patients with higher BMI, higher CD4+ T cell count, or lower baseline

139 fasting blood glucose were more likely to experience optimal immune recovery with CD4 count $\geq$

140350 cells $/ \mu \mathrm{L}$.

141

Table 3. Subject characteristics by immunological outcomes

\begin{tabular}{|c|c|c|c|}
\hline & $\begin{array}{c}\text { Suboptimal immune } \\
\text { recovery group } \\
(\mathrm{CD} 4+\text { count }<350 \\
\text { cells } / \boldsymbol{\mu L})\end{array}$ & $\begin{array}{c}\text { Immune recovery group } \\
(\mathrm{CD} 4+\text { count } \geq 350 \\
\text { cells } / \mu \mathrm{L})\end{array}$ & $p$ \\
\hline $\mathbf{N}$ & $144(36.2 \%)$ & $254(63.8 \%)$ & \\
\hline \multicolumn{4}{|l|}{ Gender(cases, ratio) } \\
\hline Male & $69(17.34 \%)$ & $120(30.15)$ & \\
\hline Age(years) & $35(32-42)$ & $35(30-40)$ & \\
\hline \multicolumn{4}{|l|}{$\begin{array}{l}\text { Marital status } \\
\text { (cases, ratio) }\end{array}$} \\
\hline Married/cohabiting & $122(84.7 \%)$ & $217(85.4 \%)$ & \\
\hline Single/divorced/widowed & $19(13.2 \%)$ & $29(11.4 \%)$ & \\
\hline Others & $3(2.2 \%)$ & $8(3.1 \%)$ & \\
\hline \multicolumn{4}{|l|}{ Routes of infection } \\
\hline IDU & $74(29.1 \%)$ & $118(46.5 \%)$ & \\
\hline Heterosexual contact & $67(26.4 \%)$ & $133(52.4 \%)$ & \\
\hline Homosexual contact & $1(0.4 \%)$ & $1(0.4 \%)$ & \\
\hline Vertical transmission & $1(0.4 \%)$ & 0 & \\
\hline Others & $1(0.4 \%)$ & $1(0.4 \%)$ & \\
\hline $\begin{array}{l}\text { Baseline HIV VL } \\
\text { (copies/mL) }\end{array}$ & $5.16(4.94-5.37)$ & $5.2(5.07-5.35)$ & \\
\hline
\end{tabular}




\begin{tabular}{|c|c|c|c|}
\hline $\begin{array}{l}\text { Baseline CD4+ count } \\
(\text { cell } / \mu \mathrm{L})\end{array}$ & $325(230-521)$ & $395(241-542)$ & \\
\hline$<200$ & $30(11.8 \%)$ & $45(17.7 \%)$ & \\
\hline $200 \leq \mathrm{CD} 4+<350$ & $49(19.3 \%)$ & $66(26.0 \%)$ & \\
\hline $350 \leq \mathrm{CD} 4+<500$ & $25(9.8 \%)$ & $62(24.4 \%)$ & \\
\hline$>500$ & $40(15.7 \%)$ & $81(31.9 \%)$ & \\
\hline $\begin{array}{l}\text { Endpoint CD4+ count } \\
(\text { cell } / \mu \mathrm{L})\end{array}$ & $231(172-290)$ & $536(413-707)$ & \\
\hline $\begin{array}{l}\text { Infection duration } \\
\text { (years) }\end{array}$ & $4.25(3.13-4.83)$ & $4.04(3.25-4.75)$ & \\
\hline ART duration & $2.30(1.58-3.88)$ & $2.46(1.71-4.04)$ & \\
\hline \multicolumn{4}{|l|}{ ART regimen } \\
\hline $\mathrm{AZT}+3 \mathrm{TC}+\mathrm{EFV}$ & $41(16.1 \%)$ & $68(26.8 \%)$ & \\
\hline $\mathrm{AZT}+3 \mathrm{TC}+\mathrm{NVP}$ & $93(36.6 \%)$ & $159(62.6 \%)$ & \\
\hline $\mathrm{TDF}+3 \mathrm{TC}+\mathrm{EFV}$ & $5(2.0 \%)$ & $10(3.9 \%)$ & \\
\hline $\mathrm{TDF}+3 \mathrm{TC}+\mathrm{NVP}$ & $1(0.4 \%)$ & $7(2.8 \%)$ & \\
\hline $\mathrm{AZT} / \mathrm{TDF}+3 \mathrm{TC}+\mathrm{LPV} / \mathrm{r}$ & $4(1.6 \%)$ & $10(3.9 \%)$ & \\
\hline HBV coinfection & $12(8.3 \%)$ & $28(11.0 \%)$ & \\
\hline HCV coinfection & $62(43.1 \%)$ & $97(38.2 \%)$ & 0.021 \\
\hline
\end{tabular}

142

143

Table 4. Factors related to suboptimal immune recovery as indicated by CD4+ T cell count

\begin{tabular}{|c|c|c|c|c|}
\hline \multirow[t]{2}{*}{ Factors } & \multicolumn{2}{|l|}{ Univariate analysis } & \multicolumn{2}{|c|}{ Multivariate analysis } \\
\hline & OR $(95 \% \mathrm{CI})$ & $p$ & OR $(95 \% \mathrm{CI})$ & $p$ \\
\hline Gender $^{\mathrm{a}}$ & $1.027(0.682-1.547)$ & 0.897 & & \\
\hline Age & $1.013(0.986-1.040)$ & 0.362 & & \\
\hline Marital status $^{\mathbf{b}}$ & $0.946(0.533-1.676)$ & 0.848 & & \\
\hline Route of infection & $0.926(0.760-1.129)$ & 0.449 & & \\
\hline Baseline HIV viral load $\left(\log _{10}\right)$ & $0.541(0.216-1.358)$ & 0.191 & & \\
\hline Baseline CD4+ T-cell count ${ }^{*}$ & $0.999(0.998-1.000)$ & 0.123 & $1.000(1.000-1.000)$ & 0.004 \\
\hline Baseline CD4/CD8 ratio & $0.845(0.424-1.684)$ & 0.632 & & \\
\hline HBV coinfection & $0.734(0.361-1.492)$ & 0.392 & & \\
\hline HCV coinfection & $1.224(0.807-1.855)$ & 0.341 & & \\
\hline Treatment Duration & $0.884(0.744-1.051)$ & 0.162 & & \\
\hline Adjustment of treatment & $1.068(0.596-1.916)$ & 0.825 & & \\
\hline BMI $^{*}$ & $0.915(0.847-0.988)$ & 0.023 & $0.921(0.854-0.994)$ & 0.034 \\
\hline Baseline total cholesterol & $1.002(0.815-1.233)$ & 0.984 & & \\
\hline Baseline triglyceride & $1.003(0.995-1.012)$ & 0.417 & & \\
\hline Baseline glucose $^{*}$ & $1.341(1.091-1.647)$ & 0.005 & $1.343(1.087-1.658)$ & 0.006 \\
\hline
\end{tabular}

Abbreviation: $\mathrm{OR}=$ Odds ratio $\mathrm{CI}=$ confidence interval.

146 a referred to males.

$147{ }^{b}$ referred to those postulated to be without fixed sexual partners, i.e. those who were single/divorced/widowed and others.

$148{ }^{*}$ Variables reaching significance in multivariate analysis.

\section{Discussion:}

151 This study regularly followed up an HIV/AIDS cohort with all patients of Yi nationality from

152 NFATP in source limited region for over two years. The patients were mostly mid-aged with

153 balanced gender distribution and mostly infected through IDU or heterosexual behaviors. At 
154 baseline, the patients were with relatively high HIV VL of around $5 \log _{10}$ copies $/ \mathrm{mL}$ and relatively

155 high CD4 counts of cells/ $\mu \mathrm{L}$. After about 2-year ART, the majority patients achieved virologic 156 treatment success (82.6\%) or complete viral suppression (65.5\%) but quite a proportion $(36.2 \%)$ of 157 patients showed suboptimal immune recovery. Longer infection duration, male gender, positive $158 \mathrm{HCV} \mathrm{Ab}$ and infection through IDU were associated with LLV while lower BMI and higher fasting 159 blood glucose were associated with suboptimal CD4 count recovery after viral suppression. To our 160 knowledge, this study is one of the few cohort studies with long-term follow-up in this unique 161 population, with interventions and surveillances in strict compliance with the national policy and 162 with only government financed resources as far as possible. Thus, this study delineates detailed 163 medical profiles of PLWH in Liangshan under management in NFATP and provides clues to 164 maximize patients' clinical benefit without aggravating personal or national burden.

165 Viral suppression is the hallmark of HIV treatment success as well as optimal long-term 166 prognosis. While definite viral failure, generally resulting from drug-resistant mutation, requires 167 regimen modification, the grey zone of the persistent LLV remains a controversial issue concerning 168 its clinical consequences and management. There were slightly more patients observed with LLV in 169 this cohort than in other studies $(16.9 \%$ vs $15.7 \%)(10)$. The speculation that LLV foreshadows 170 subsequent viral failure is quite reasonable. Evidence from several studies has corroborated that 171 LLV is strongly associated with viral failure with the HR of around 3.14-3.97 (11-14). But findings 172 concerning the impact of LLV on clinical outcomes such as AIDS events and mortality failed to 173 reach a consensus even in these studies. There are also studies reporting no association between 174 transient LLV and adverse virologic and immunological outcomes(15-17). In this study, patients 175 with LLV on ART displayed significantly lower CD4 count compared with patients with viral 176 suppression at endpoint ( $402[278,598]$ vs $365[235,472], p=0.024)$. There is the possibility that 177 longer follow-up are required to explicitly disclose the influence of LLV on clinical outcomes. 178 Besides, for such observations, more frequent monitoring such as every 3 or 6 months should be 179 necessary to more aptly delineate the VL dynamics during ART.

180 Concerning the mechanism of LLV, a recent study has reported that most viral non-suppression 181 episodes [173/1935 (8.9\%)] were attributable to nonadherence in $30 \%$ and to pre-treatment drug 
182 resistance (PDR) to NRTIs in 10\% (18). Notably, at contemporary PDR prevalence of 10-25\%,

183 PDR would explain $13-30 \%$ of viral non-suppression(18). PDR has been one of the epidemic 184 characteristics in Liangshan area. A survey in 2017 unraveled a PDR rate of $12.2 \%$ in this area, 185 reported as the highest in China, and the subgroup of injecting drug users showed significantly 186 higher PDR(19). In our analysis, infection through IDU (OR =1.584 [95\%CI: 1.022-2.455], $p=0.04$ ) 187 is associated with higher risk of LLV. It's reasonable to speculate that transmission of PDR viral 188 subtypes through IDU have contributed to relatively high prevalence of LLV. It's even more 189 worrying that previous study reported 2.03-fold higher prevalence of PDR among patients 190 diagnosed from 2017-2018 than that among patients diagnosed from 2009-2016, suggesting a 191 rapidly growing PDR prevalence over time(95\% CI: 1.18-5.76; $\mathrm{P}=0.018)(20)$. Rigorous actions to 192 control illegal drug deal and education on the pernicious influence of IDU are in urgent need to 193 improve the HIV epidemics in this area. Male gender (OR $=1.632$ [95\%CI: 1.053-2.53], $p=0.028$ ) 194 is another risk factor of unsatisfactory CD4 count recovery identified in this study. It's widely 195 accepted that $\geq 95 \%$ adherence to antiretroviral medications is required for optimal treatment effect.

196 The viral suppression rate was reported to be $81 \%$ in patients with a $>95 \%$ treatment compliance 197 and dramatically decreased to $64 \%$ in those with $90 \sim 95 \%$ compliance(21). One study looking into 198 self-management of PLWH in Liangshan area has found lower overall self-management scores and 199 an even lower score in male patients, indicating poor adherence to ART medications(22). Moreover, 200 a global survey revealed more IDU in men than in women in south Asia(23), which could also be 201 the case in Liangshan area. Patient education and supervision should be strengthened to promote 202 better self-management for improved long-term prognosis. Therefore, the unique characteristic of 203 injecting drug users contributes a lot to the less satisfying treatment outcomes in Liangshan area.

204 Closer monitoring of drug resistance, more rigorous treatment, pre-treatment drug resistance tests 205 are potential approaches for better therapeutic effectiveness in this area.

206 Immune recovery as indicated by CD4 count increase or absolute CD4 count is another aim of 207 HIV healthcare. After around 2-year ART, 63.8\% of the patients with viral suppression achieved 208 optimal immune recovery of $\geq 350$ cells $/ \mu \mathrm{L}$ in our cohort with the remaining displaying 209 unsatisfactory immunological outcomes. However, the ART duration in some patients have not 
210 reached 2 years at the endpoint, which is one of the criteria for immunological non-response

211 proposed by one study after comprehensive comparison of different definitions across studies(24).

212 Thus, these patients might retain the chance of experiencing optimal immune after prolonged ART.

213 At the endpoint of our study, per the criteria of an absolute CD4+ T-cell count $<350$ cells $/ \mu \mathrm{L}$ after

$214 \geq 24$ months of ART with viral suppression, there were 48 (18.1\%) patients determined as immune

215 non-responders, which is comparable to that reported in other studies (10-40\%, depending on the

216 definitions and cohorts)(25). Suboptimal immune recovery has long been proved to be associated

217 with increased mortality and morbidity (26-29). Risk factors for this suboptimal response to

218 treatment include older age, lower nadir CD4 count, coinfection with HBV/HCV/CMV(30, 31). The

219 analysis of this cohort revealed factors related to suboptimal immune recovery less frequently

220 reported in other studies, including BMI and fasting blood glucose. Although both indicators of the

221 study cohort were within normal range, most HIV infected patients in Liangshan are known to be

222 weakened by malnutrition, which would exacerbate metabolic disorders and accelerate hyper-

223 catabolism(32-34). A randomized controlled trial reported that nutritional deficiency hampers CD4+

$224 \mathrm{~T}$ cell recovery even during antiretroviral therapy(34) and higher BMI has been found to be

225 significantly associated with long-term advantages in immune recovery on ART(35). Nutritional

226 status assessment might be included in routine management of PLWH in this area for a better

227 immunological response. Meanwhile, it seems paradoxical that higher fasting blood glucose within

228 normal range is related to suboptimal immune recovery in our analysis. PLWH experience a 2- to

229 4-fold greater incidence of elevated fasting glucose or hyperinsulinemia than the general

230 population(36). Glucose metabolic disorders together with other non-AIDS comorbidities is

231 associated with chronic systemic inflammation and residual immune cell activation, which are

232 known as status related to suboptimal immune recovery(37). A study of HIV+ patients with impaired

233 glucose tolerance defined impaired fasting glucose as 5.56-6.94 mmol/L(38), which is comparable

234 to that $(4.99 \pm 1.01 \mathrm{mmol} / \mathrm{L})$ of patients in our cohort. It's reasonable to interpret our patients as with

235 impaired fasting glucose, especially when the patients' lower BMI is taken into consideration. Under

236 this speculation, higher fasting blood glucose indicates poor glucose tolerance with pro-

237 inflammatory effect, further contributing to poor immune recovery. 
Although the detailed profiles of HIV patients from NFATP delineated in this study could

239 provide important clues to better deployment of HIV management for achieving the 90-90-90 targets,

240 there are certain limitations. The follow-up period was not long enough to observe long-term

241 outcomes of these patients and the information obtained merely from observation was inadequate

242 to delve into the underlying mechanisms in issues such as LLV and suboptimal immune recovery.

243 Based on evidence from this study, elaborately-designed trial with more frequent monitoring and

244 drug resistant mutation detection is expected to provide more evidence to guide better HIV 245 management in Zhaojue county, Liangshan Prefecture.

\section{Conclusions}

247 PLWH in Zhaojue County, Liangshan is a unique population with high prevalence of IDU and 248 HIV-HBV/HCV coinfection, which is associated with suboptimal immune recovery despite 249 satisfactory virologic outcomes after ART. The majority achieved virologic treatment success but 250 improvement is in urgent need for patients with LLV and those with suboptimal immune recovery. 251 Close monitoring, integrated management including nutrition status management and treatment 252 compliance education and stringent control of illegal drug deal by relevant local authorities should 253 be conducive.

\section{Methods}

\section{Study aim and design}

256 This is a retrospective cohort study aimed to assess the clinical outcomes of PLWH from 257 NFATP and explore risk factors associated with suboptimal outcomes, so as to provide clues for 258 better better HIV management in Zhaojue county, Liangshan Prefecture.

\section{Subject enrollment}

260 NFATP provides free ART as well as free routine follow-up tests, including hematology, 261 urinalysis and blood liver function every 6 months; renal function for patients taking tenofovir 262 disoproxil fumarate (TDF) and serum lipid for patients taking lopinavir/ritonavir (LPV/r) every 6 263 months; CD4+ count and HIV viral load (VL) every year. A national data system has been 264 established for NFATP, designed to record comprehensive patient profiles including sociodemographics, baseline medical condition and follow-up data. Considering the local 
266 implementation of relevant policies and that the changed clinical practice after the release of 267 guidelines for HIV prevention and control, the $3^{\text {rd }}$ version, patients from NFATP who initiated ART 268 between January 1, 2015 and December 31, 2017 were screened. Patients were included who i) were $269 \geq 18$ years old at ART initiation; ii) had confirmed HIV diagnosis by Western Blot; iii) had routine 270 lab tests performed; iv) had follow-up records of test results available. The baseline was the date of 271 recorded ART initiation and the endpoint was defined as December 31, 2019, death, regimen 272 switched to charged options because of personal choice or medical needs, whichever came first. The 273 details of patient screening are shown in Figure 1.

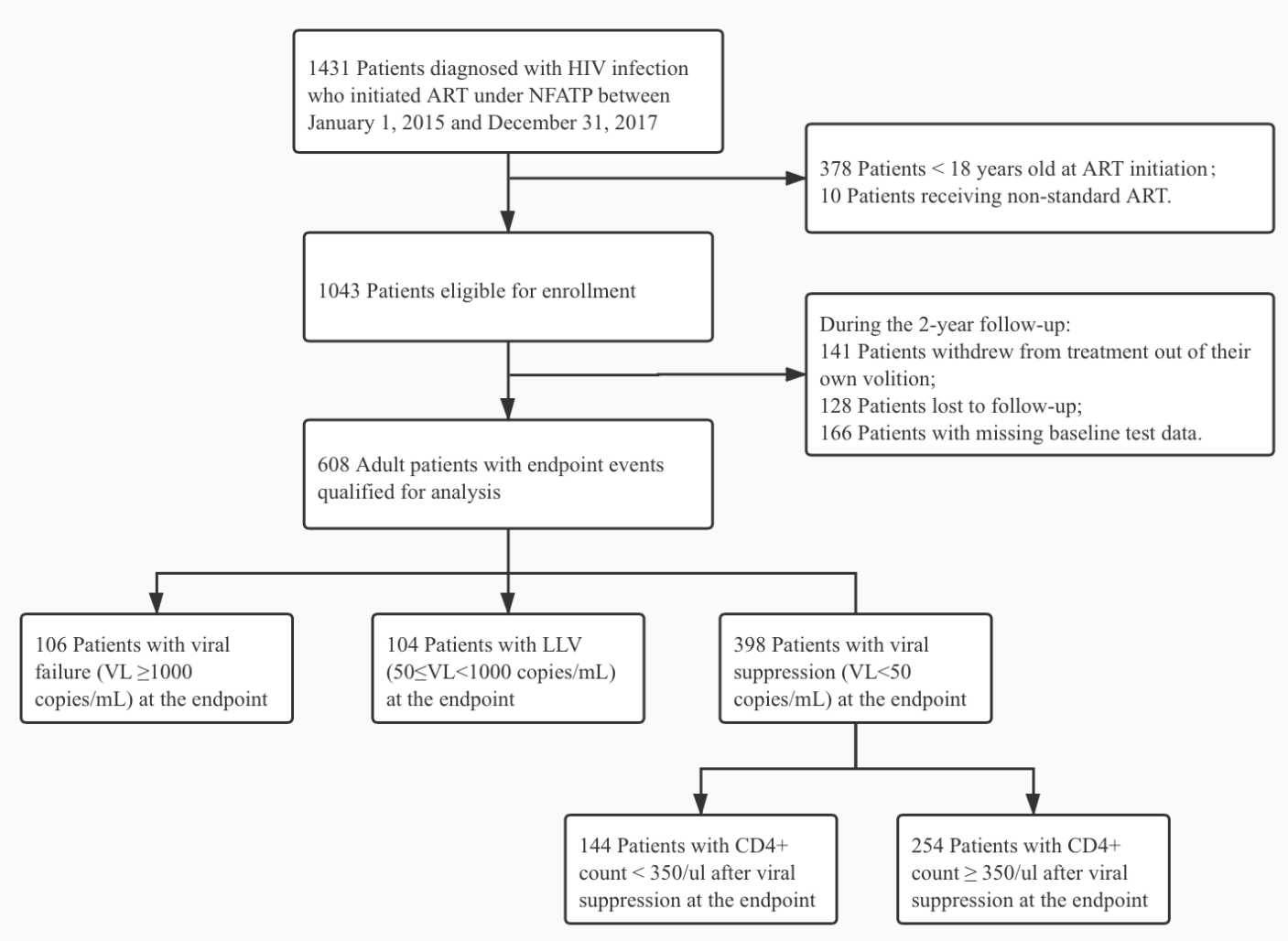

Figure 1 Flowchart of subject enrollment.

Variables, definitions and outcomes

The definitions of some terminology varies across various studies due to different detection techniques and study population. The definitions to be clarified in this study include:

- Viral suppression was defined as the viral load $<50$ copies $/ \mathrm{mL}$, viral failure was defined as the viral load $\geq 1000$ copies $/ \mathrm{mL}$ and low-level viremia (LLV) was defined as the viral load of 50-999 copies/mL after 6 months of ART; HIV/AIDS (2018) is a viral load persistently $>200$ copies $/ \mathrm{mL}$ after 1 year of ART. But 
considering the local circumstances, the lower limit of 1000 copies $/ \mathrm{mL}$ in WHO guidelines, 2016 is adopted.

- Immune recovery is the most controversial terms differing greatly across studies. Generally, immune recovery is measured as absolute CD4+ count or the absolute or

\section{Statistics} percentage increase of CD4+ count with suppressed viral replication after certain period of ART(39). The cutoff values of CD4+ count for optimal and suboptimal immune recovery are 200, 250, 350, 400 and 500 cells $/ \mu \mathrm{L}$ and the duration of ART varies from 1 to 12 years across studies. In this study, the relatively well-recognized criteria was adopted as suboptimal immune recovery, that is, $\mathrm{CD} 4+$ count $<350$ cells $/ \mu \mathrm{L}$ at endpoint with $\mathrm{VL}$ $<50$ copies.

- Regimen switch. Six out of all the free antiretroviral regimens were applied in this area, namely TDF + lamivudine (3TC) + efavirenz (EFV), zidovudine (AZT) + 3TC + EFV, $\mathrm{AZT}+3 \mathrm{TC}+$ nevirapine $(\mathrm{NVP}), \mathrm{AZT}+3 \mathrm{TC}+\mathrm{LPV} / \mathrm{r}, \quad \mathrm{TDF}+3 \mathrm{TC}+\mathrm{NVP}$ and $\mathrm{TDF}+3 \mathrm{TC}+\mathrm{LPV} / \mathrm{r}$. Regimen switch between these regimens after ART initiation were recorded regardless of the rationale for switch.

- The main outcomes of the analysis were the rate of viral suppression and optimal immune recovery at endpoint and the secondary outcomes were the risks factors associated with suboptimal immune recovery at endpoint.

304 New York, US). The category variables were described as number and percent and analyzed with 305 chi-square test. The continuous variables were described as median and interquartile range (IQR) or 306 mean and standard deviation (SD) and analyzed by $U$ test or $t$ test as appropriate. Univariate and 307 multivariate log-binomial regression models were used to assess determinants of suboptimal 308 immune recovery, producing odds ratios (ORs) and confidence intervals (CIs). All $p$-values were 2309 sided, and $p<0.05$ was considered statistically significant.

\section{$310 \quad$ List of abbreviations}

$\begin{array}{ll}\text { 3TC } & \text { Lamivudine } \\ \text { AIDS } & \text { Acquired immunodeficiency syndrome } \\ \text { ART } & \text { Antiretroviral treatment } \\ \text { AZT } & \text { Zidovudine } \\ \text { BMI } & \text { Body mass index } \\ \text { CI } & \text { Confidence interval } \\ \text { EFV } & \text { Efavirenz }\end{array}$




\begin{tabular}{|c|c|}
\hline HBsAg & Hepatitis B virus surface antigen \\
\hline HBV & Hepatitis B virus \\
\hline $\mathrm{HCV}$ & Hepatitis $\mathrm{C}$ virus \\
\hline HIV & Human immunodeficiency virus \\
\hline IDU & Injecting drug use \\
\hline LLV & Low level viremia \\
\hline $\mathrm{LPV} / \mathrm{r}$ & Lopinavir/ ritonavir \\
\hline NFATP & National Free Antiretroviral Treatment Program \\
\hline NNRTI & Non-nucleotide reverse transcriptase inhibitor \\
\hline NRTI & Nucleotide reverse transcriptase inhibitor \\
\hline NVP & Nevirapine \\
\hline OR & Odds ratio \\
\hline PDR & Pre-treatment drug resistance \\
\hline PLWH & People living with HIV \\
\hline $\mathrm{SD}$ & Standard deviation \\
\hline TDF & Tenofovir disoproxil fumarate \\
\hline VL & Viral load \\
\hline
\end{tabular}

\section{Declarations}

\section{Ethics approval and consent to participate}

314 This study was approved by the Medical Ethics Committee of West China Hospital of Sichuan

315 University and written informed consents were acquired from all participants (Annual Audit No.

316450 , Version 2020.5).

317 Consent for publication

318 Not applicable.

\section{Availability of data and materials}

320 The data used and/or analyzed during the current study are available from the corresponding 321 author on reasonable request. 


\section{Funding}

This study was supported by the 1.3.5 project for disciplines of excellence, West China Hospital,

326 Sichuan University (No. ZYGD20009).

\section{Authors' contributions}

HT and LB proposed the conception and designed the study. LC, LD, SK, FM and CL

implemented the study and collected the data. LC and SK analyzed the data. SK drafted the manuscript. LB and HT revised the manuscript. HT provided fund support and approved the

331 submitted version

Acknowledgments

Not applicable.

\section{References:}

336 1. Ding Y, Ma Z, He J, Xu X, Qiao S, Xu L, et al. Evolving HIV Epidemiology in 337 Mainland China: 2009-2018. Curr HIV/AIDS Rep. 2019;16(6):423-30.

338 2. Prevention NCfASCa, Prevention CfDCa. Annals of information on 339 comprehensive prevention and treatment for AIDS, STD and hepatitis C. 2018.

340 3. Ma Y, Dou Z, Guo W, Mao Y, Zhang F, McGoogan JM, et al. The Human 341 Immunodeficiency Virus Care Continuum in China: 1985-2015. Clin Infect Dis. 342 2018;66(6):833-9.

343 4. Zhao QG, Gong YH, Liao Q, Yu G, Wang K, Wang J, et al. [Estimation on the HIV3441 incidence in Liangshan Yi Autonomous Prefecture, under BED-capture enzyme 345 immunoassay, from 2011 to 2013]. Zhonghua Liu Xing Bing Xue Za Zhi. 346 2016;37(8):1105-7.

347 5. Jiang X, Xu JL, Jike KCN, Yu G, Yu HL, Wang J, et al. [Epidemiological analysis 348 of the deaths with antiretroviral treatment among adult HIV/AIDS patients in 349 Liangshan Yi Autonomous Prefecture from 2005 to 2015]. Zhonghua Liu Xing Bing $350 \quad$ Xue Za Zhi. 2019;40(9):1116-9.

351 6. Yuan D, Liu M, Jia P, Li Y, Huang Y, Ye L, et al. Prevalence and determinants of 352 virological failure, genetic diversity and drug resistance among people living with HIV 353 in a minority area in China: a population-based study. BMC Infect Dis. 2020;20(1):443.

354 7. Liang X, Bi S, Yang W, Wang L, Cui G, Cui F, et al. Epidemiological serosurvey 355 of hepatitis B in China--declining HBV prevalence due to hepatitis B vaccination. 356 Vaccine. 2009;27(47):6550-7. 
8. Cui Y, Jia J. Update on epidemiology of hepatitis B and C in China. J Gastroenterol Hepatol. 2013;28 Suppl 1:7-10.

359 9. Xie J, Han Y, Qiu Z, Li Y, Li Y, Song X, et al. Prevalence of hepatitis B and C 360 viruses in HIV-positive patients in China: a cross-sectional study. J Int AIDS Soc. $361 \quad 2016 ; 19(1): 20659$.

362 10. Elvstam O, Medstrand P, Yilmaz A, Isberg PE, Gisslen M, Bjorkman P. Virological 363 failure and all-cause mortality in HIV-positive adults with low-level viremia during 364 antiretroviral treatment. PLoS One. 2017;12(7):e0180761.

365 11. Bernal E, Gomez JM, Jarrin I, Cano A, Munoz A, Alcaraz A, et al. Low-Level 366 Viremia Is Associated With Clinical Progression in HIV-Infected Patients Receiving 367 Antiretroviral Treatment. J Acquir Immune Defic Syndr. 2018;78(3):329-37.

368 12. Joya C, Won SH, Schofield C, Lalani T, Maves RC, Kronmann K, et al. Persistent 369 Low-level Viremia While on Antiretroviral Therapy Is an Independent Risk Factor for 370 Virologic Failure. Clin Infect Dis. 2019;69(12):2145-52.

371 13. Vandenhende MA, Perrier A, Bonnet F, Lazaro E, Cazanave C, Reigadas S, et al. 372 Risk of virological failure in HIV-1-infected patients experiencing low-level viraemia 373 under active antiretroviral therapy (ANRS $\mathrm{C} 03$ cohort study). Antivir Ther. 374 2015;20(6):655-60.

375 14. Havlir DV, Bassett R, Levitan D, Gilbert P, Tebas P, Collier AC, et al. Prevalence 376 and predictive value of intermittent viremia with combination hiv therapy. JAMA. 377 2001;286(2):171-9.

378 15. Eastburn A, Scherzer R, Zolopa AR, Benson C, Tracy R, Do T, et al. Association 379 of low level viremia with inflammation and mortality in HIV-infected adults. PLoS One. $380 \quad$ 2011;6(11):e26320.

381 16. Quiros-Roldan E, Raffetti E, Castelli F, Foca E, Castelnuovo F, Di Pietro M, et al. 382 Low-level viraemia, measured as viraemia copy-years, as a prognostic factor for 383 medium-long-term all-cause mortality: a MASTER cohort study. J Antimicrob 384 Chemother. 2016;71(12):3519-27.

385 17. Garcia-Gasco P, Maida I, Blanco F, Barreiro P, Martin-Carbonero L, Vispo E, et al. 386 Episodes of low-level viral rebound in HIV-infected patients on antiretroviral therapy: 387 frequency, predictors and outcome. J Antimicrob Chemother. 2008;61(3):699-704.

388 18. Inzaule SC, Bertagnolio S, Kityo CM, Siwale M, Akanmu S, Wellington M, et al. 389 The relative contributions of HIV drug resistance, nonadherence and low-level viremia 390 to viremic episodes on antiretroviral therapy in sub-Saharan Africa. AIDS. $391 \quad 2020 ; 34(10): 1559-66$.

392 19. Kang RH, Liang SJ, Ma YL, Liang S, Xiao L, Zhang XH, et al. Pretreatment HIV 393 drug resistance in adults initiating antiretroviral therapy in China, 2017. Infect Dis 394 Poverty. 2020;9(1):54.

395 20. Liu L, Dong A, Liao L, Feng Y, Shao Y, Liang S, et al. Survey of Pretreatment HIV 396 Drug Resistance and Genetic Transmission Network Analysis Among HIV Patients in 397 a High Drug-Use Area of Southwest China. Curr HIV Res. 2019;17(6):441-51.

398 21. Paterson DL, Swindells S, Mohr J, Brester M, Vergis EN, Squier C, et al. 
Adherence to protease inhibitor therapy and outcomes in patients with HIV infection. Ann Intern Med. 2000;133(1):21-30.

22. Wang H, Chen AC, Wan S, Chen H. Status and associated factors of selfmanagement in people living with HIV/AIDS in Liangshan area, China: a crosssectional study. Patient Prefer Adherence. 2019;13:863-70.

23. Degenhardt L, Peacock A, Colledge S, Leung J, Grebely J, Vickerman P, et al. Global prevalence of injecting drug use and sociodemographic characteristics and prevalence of HIV, HBV, and HCV in people who inject drugs: a multistage systematic review. Lancet Glob Health. 2017;5(12):e1192-e207.

24. Rb-Silva R, Goios A, Kelly C, Teixeira P, Joao C, Horta A, et al. Definition of Immunological Nonresponse to Antiretroviral Therapy: A Systematic Review. J Acquir Immune Defic Syndr. 2019;82(5):452-61.

25. Nakanjako D, Kiragga AN, Musick BS, Yiannoutsos CT, Wools-Kaloustian K, Diero L, et al. Frequency and impact of suboptimal immune recovery on first-line antiretroviral therapy within the International Epidemiologic Databases to Evaluate AIDS in East Africa. AIDS. 2016;30(12):1913-22.

26. Massanella M, Negredo E, Clotet B, Blanco J. Immunodiscordant responses to HAART--mechanisms and consequences. Expert Rev Clin Immunol. 2013;9(11):113549.

27. Lewden C, Chene G, Morlat P, Raffi F, Dupon M, Dellamonica P, et al. HIVinfected adults with a CD4 cell count greater than 500 cells $/ \mathrm{mm} 3$ on long-term combination antiretroviral therapy reach same mortality rates as the general population. J Acquir Immune Defic Syndr. 2007;46(1):72-7.

28. Antiretroviral Therapy Cohort C. Life expectancy of individuals on combination antiretroviral therapy in high-income countries: a collaborative analysis of 14 cohort studies. Lancet. 2008;372(9635):293-9.

29. Engsig FN, Gerstoft J, Kronborg G, Larsen CS, Pedersen G, Roge B, et al. Longterm mortality in HIV patients virally suppressed for more than three years with incomplete CD4 recovery: a cohort study. BMC Infect Dis. 2010;10:318.

30. Collazos J, Valle-Garay E, Carton JA, Montes AH, Suarez-Zarracina T, De la Fuente B, et al. Factors associated with long-term CD4 cell recovery in HIV-infected patients on successful antiretroviral therapy. HIV Med. 2016;17(7):532-41.

31. Kroeze S, Ondoa P, Kityo CM, Siwale M, Akanmu S, Wellington M, et al. Suboptimal immune recovery during antiretroviral therapy with sustained HIV suppression in sub-Saharan Africa. AIDS. 2018;32(8):1043-51.

32. Fajardo-Rodriguez A, Lara del Rivero-Vera CM. [Nutritional intervention in HIV/AIDS: practical guide for its implementation and follow-up]. Gac Med Mex. 2001;137(5):489-500.

33. Roubenoff R. The pathophysiology of wasting in the elderly. J Nutr. 1999;129(1S Suppl):256S-9S.

34. Sun PT, Yu W, Li TS, Lin Q, Guo FP, Zhou XH, et al. Association of Body Composition and Other Clinical Factors with Incomplete Immune Response after 
441 Highly Active Antiretroviral Therapy. Zhongguo Yi Xue Ke Xue Yuan Xue Bao. 442 2017;39(4):459-64.

443 35. Koethe JR, Jenkins CA, Lau B, Shepherd BE, Wester W, Rebeiro PF, et al. Higher 444 Time-Updated Body Mass Index: Association With Improved CD4+ Cell Recovery on 445 HIV Treatment. J Acquir Immune Defic Syndr. 2016;73(2):197-204.

446 36. Brown TT, Li X, Cole SR, Kingsley LA, Palella FJ, Riddler SA, et al. Cumulative 447 exposure to nucleoside analogue reverse transcriptase inhibitors is associated with 448 insulin resistance markers in the Multicenter AIDS Cohort Study. AIDS. $4492005 ; 19(13): 1375-83$.

450 37. Wada NI, Jacobson LP, Margolick JB, Breen EC, Macatangay B, Penugonda S, et 451 al. The effect of HAART-induced HIV suppression on circulating markers of 452 inflammation and immune activation. AIDS. 2015;29(4):463-71.

453 38. Best C, Struthers H, Laciny E, Royal M, Reeds DN, Yarasheski KE. Sitagliptin 454 Reduces Inflammation and Chronic Immune Cell Activation in HIV+ Adults With 455 Impaired Glucose Tolerance. J Clin Endocrinol Metab. 2015;100(7):2621-9.

456 39. Yang X, Su B, Zhang X, Liu Y, Wu H, Zhang T. Incomplete immune reconstitution 457 in HIV/AIDS patients on antiretroviral therapy: Challenges of immunological non458 responders. J Leukoc Biol. 2020;107(4):597-612. 


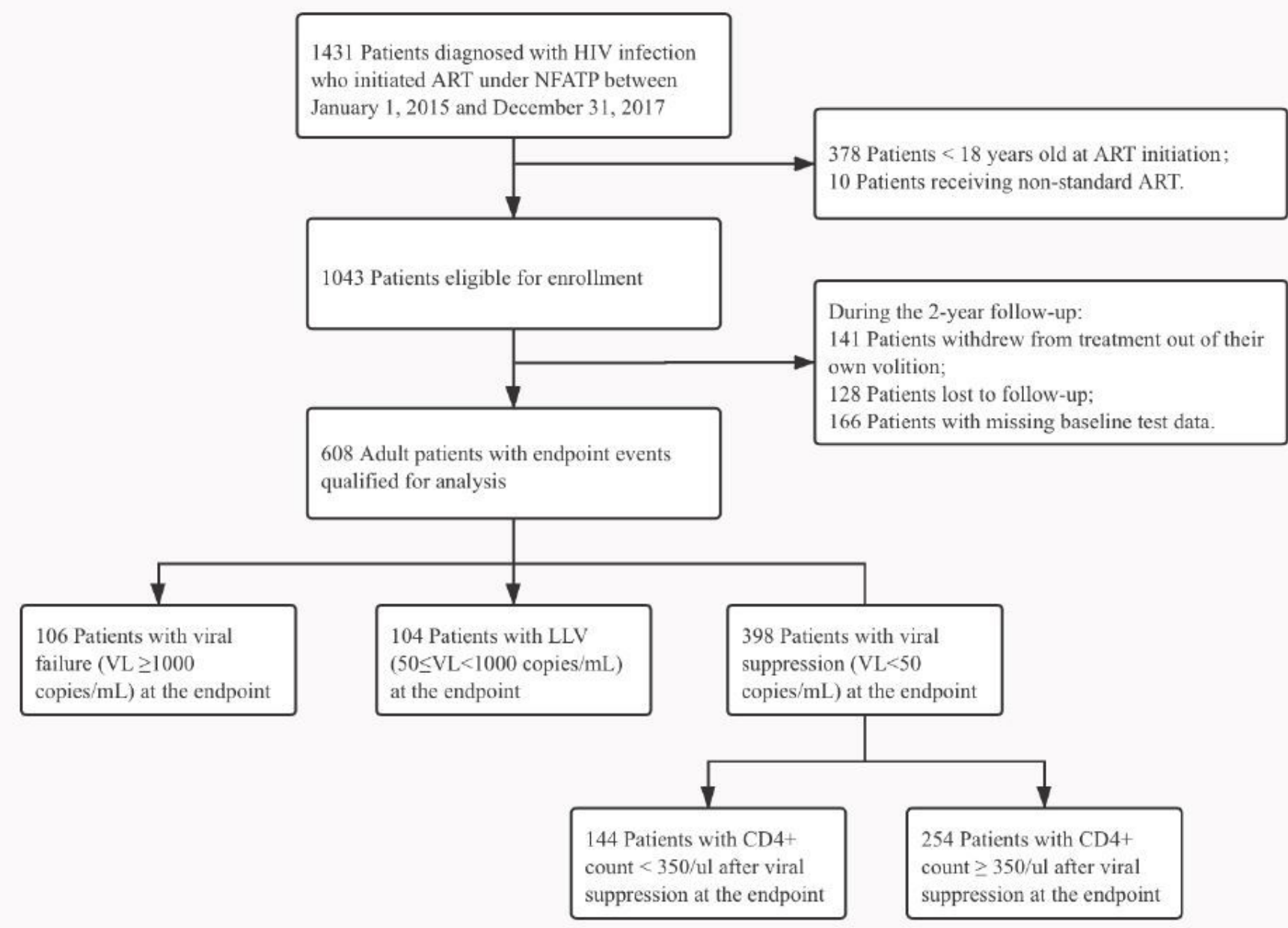

Figure 1

Flowchart of subject enrollment. 